\title{
Factors That Influence Professional Quality of Life (Pro-QOL) on Clinical Nurses
}

\author{
Febrina Secsaria Handini, Fitriyanti Patarru', Basilius Yosepfus Weu, Heryyanoor and Sena \\ Wahyu Purwanza
}

Faculty of Nursing, Universitas Airlangga, Surabaya, Indonesia

\begin{abstract}
Introduction: Nurses are professionals that work by involving cognitive and emotional aspects simultaneously when providing nursing care, which provides both positive and negative experiences. These experiences can affect nurses' professional quality of life (Pro-QOL). Professional quality of life has three dimensions, namely burnout (BO), compassion satisfaction (CS) and Secondary Traumatic Stress (STS). This paper presents a systematic review of the literature that examined factors that influence the professional quality of life on clinical nurses.

Methods: Articles are obtained from the Scopus, Science Direct and Emerald databases using keywords Compassion Satisfaction, Burnout, Secondary Traumatic Stress, Nurse. The search identified 69 articles and 15 relevant research articles published between 2014-2019.

Results: The results show that the inability of nurses to control the core of self-evaluation and the five-factor model (FFM) of personality traits, high workload, pressure in the work and imbalance between rewards and work, coping nurses and poor emotional support, rejection and giving up behavior can increase the number of those with compassion fatigue.

Conclusion: The results show that the inability of nurses to control the core of self-evaluation and the five-factor model (FFM) of personality traits, high workload, pressure in the work and imbalance between rewards and work, coping nurses and poor emotional support, rejection and giving up behavior can increase the number of those with compassion fatigue.
\end{abstract}

\section{ARTICLE HISTORY}

Received: Dec 26, 2019

Accepted: Dec 31, 2019

\section{KEYWORDS}

self foot care; foot care behavior; diabetic foot; diabetes mellitus

\section{CONTACT}

Febrina Secsaria Handini $\triangle$ febrina.secsaria.handini2018@fkp.unair.ac.id

$\doteq$ Faculty of Nursing, Universitas Airlangga, Surabaya, Indonesia

Cite this as: Handini, F.S., Patarru', F., Weu, B.Y., Heryyanoor, H., \& Purwanza, S.W.. (2019). Factors That Influence Professional Quality of Life (Pro-QOL) on Clinical Nurses. Jurnal Ners, 14(3si), 393-396. doi:http://dx.doi.org/10.20473/jn.v14i3(si).17176

\section{INTRODUCTION}

Clinical nurses often work in environments that are full of pressure and where the workload is quite high, because each day they face complex and different patient conditions. For example, professional care providers in cancer palliative care. Nurses who provide professional care to patients with advanced cancer face challenges and stress because nurses must provide holistic care in the physical, psychological, spiritual, and environmental aspects (Kaur, Sharma, \& Chaturvedi, 2018). This can be a source of psychological stress for nurses and makes them vulnerable to burnout and/or compassion fatigue (Kaur et al., 2018). Burnout and compassion fatigue is an aspect of professional quality of life (ProQOL).
The professional quality of life (Pro-QOL) is the quality perceived by a professional related to work as a service provider. There are two aspects in professional quality of life (Pro-QOL), namely Compassion Satisfaction (CS) as a positive aspect and Compassion Fatigue (CF) as a negative aspect (B.H. Stamm, 2010). The nurse is able to feel satisfaction after helping others and is able to do a good job, so that satisfaction is called Compassion Satisfaction. This satisfaction is a positive perspective and can improve the ability of nurses to care for patient (Stamm, 2016. Bibliography et al., 2016). Compassion Fatigue (CF) is a negative consequence of taking care of patients (Figley, 2018). Compassion Fatigue has two aspects, namely burnout (BO) and Secondary Traumatic Stress (STS) (B.H. Stamm, 2010). Secondary traumatic stress refers to emotional 
responses or behavior caused by exposure to other people's traumatic experiences. Burnout is a negative emotional reaction to external stressors that are connected to the work environment.

Based on the description of the background, the purpose of the current study was to systematically review and synthesize the factors related to or which influence the professional quality of life (Pro-QOL).

\section{MATERIALS AND METHODS}

\section{Search strategies}

The search strategy involved a review of three databases: Scopus, Science Direct and Emerald using keywords "burnout", "satisfaction compassion", "secondary traumatic stress", and "nurse". The search included English language articles published between 2014-2019.

\section{Selection Criteria}

Article searches are conducted from November 2018 to March 2019. Articles were selected and included if they met the following criteria 1) the study about professional quality of life in clinical nurses; 2) was original research; 3) were studies written in English; 4) the location of the study is a hospital or a health center; 5) research articles published between 20142019. An article was excluded if it was a thesis or part of conference proceedings.

\section{Data Extraction}

For articles that fulfill the inclusion and exclusion criteria for fifteen articles, we extracted the author, year, study design, sample, measurement, analysis, and whether the results of the study were significant or not significant. In addition, we extracted the results of articles that discuss factors related to or are influenced by professional quality of life (Pro-QOL). Differences or disagreement in research results are resolved through discussion with all authors.

\section{RESULTS}

\section{Selection of Studies}

The search resulted in a total of 244 studies: 69 from Scopus, 13 from Emerald, 162 from Science Direct. Of these, 15 manuscripts were relevant to factors that are related to or are influenced by professional quality of life (Pro-QOL) and which were screened using inclusion criteria. Fifteen studies were maintained after screening quality assessment.

\section{Study Methods}

From the results of the study selection, we found 15 study respondents. Studies are limited to between 2014-2019. Two studies were published in 2014, two studies were published in 2015, two studies were published in 2016, two studies were published in 2017, six studies were published in 2018, and one study was published in 2019 . Based on the study design, all respondents used a quantitative research approach with cross-sectional details of 7
Search term:

Compassion Satisfaction, AND Burnout AND Secondary Traumatic Stress AND Nurse

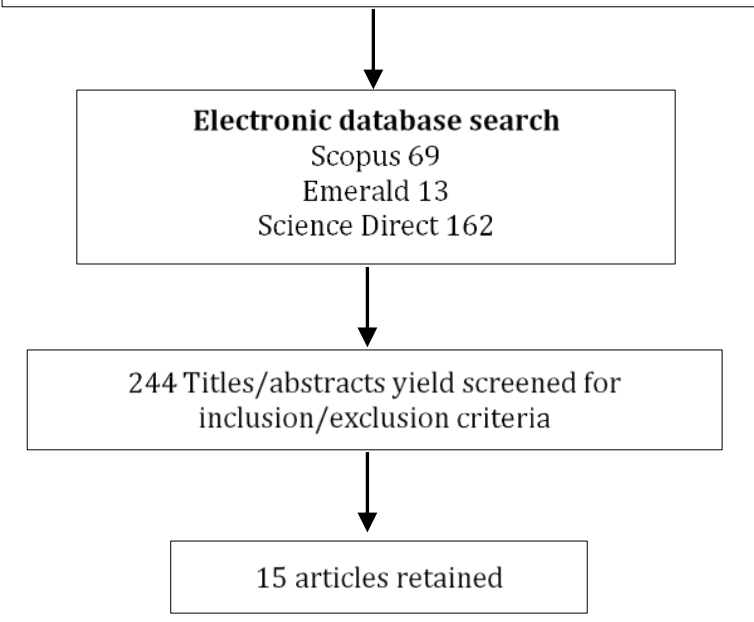

Figure 1: Literature search flow

respondents, a cross-sectional descriptive of 3 respondents, a cross-sectional survey of 4 respondents and a non-experimental longitudinal design of 1 respondent. Regarding the research subjects, the following data were obtained: 12 respondents were nurses and/or nurse assistants who worked in hospitals or health care centers such as trauma centers, 2 health professionals and 1 respondent were pregnancy termination providers. For data sources, in addition to socio-demographic information, fifteen articles used questionnaires as instruments. Thirteen of the fifteen articles used Professional Quality of Life Questionnaire Version 5 (Pro QOL V) (Amin, Vankar, Nimbalkar, \& Phatak, 2015; Barr, n.d., 2018; Foster, 2019; Friedmann et al., 2014; Hegney, Desley G; Craigie, Mark; Hemsworth, 2014; Hegney, Rees, Eley, Osseiran-moisson, \& Rees, 2015; Jang, Kim, \& Kim, 2016; Kaur et al., 2018; C. F. Rn \& Rn, 2017; S. J. Rn, Hons, Rn, \& Rn, 2017; Teffo, Levin, \& Rispel, 2018; Wu, Singh-carlson, Odell, Reynolds, \& Su, 2016), one article used the Copenhagen Burnout Inventory (CBI) (Hämmig, 2018), and one article used the Secondary Traumatic Stress Scale (Kellogg, Knight, Dowling, \& Crawford, 2018). In addition to a questionnaire to measure professional quality of life, there are several questionnaires that are used as measuring instruments to explore factors that are related to, or influence, professional quality of life, such as the Core Self-Evaluation Scale, Big-Five Inventory (BFI), Positive and Negative Affect Schedule, Copenhagen Psychosocial Questionnaire (COPSOQ), Perceived Stress Scale (PSS14), The Chinese Social Support Questionnaire, Depression Anxiety Stress Scale (DASS 21-short form), a modified Hall's Professionalism Inventory (HPI) Scale, The Brief COPE, The MarloweCrowne Social Desirability-Short Form, Spielberger State-Trait Anxiety Inventory form Y2 (STAI-Y2), and the Connor-Davidson Resilience Scale (CD-RISC25). 


\section{DISCUSSION}

Professional quality of life is divided into two aspects, namely the positive aspect called compassion satisfaction (CS) and the negative aspect called compassion fatigue (CF), which consists of two things, burnout (BO) and secondary traumatic stress (STS). The results of the study by Kaur et al. (2018) show that nurses are one of the professional health workers who have a higher level of burnout and secondary traumatic stress than the other professional health workers (Kaur et al., 2018). From the results of the analysis of several literature studies above, it was found that several factors influence the positive and negative aspects in the professional quality of life in clinical nurses.

Increasing compassion satisfaction of a nurse can be influenced by several factors, including: nurses' ability to regulate core self-evaluation (self-esteem, self-efficacy, emotional stability, locus of control) and the five-factor model (FFM) of personality traits that includes neuroticism, agreeableness, extraversion, conscientiousness, and openness (Barr, 2018), high professionalism at work (Jang et al., 2016), and high educational qualifications (S. J. Rn et al., 2017; Wu et al., 2016). Nurses who have the ability to build social relationships, especially with colleagues, have a positive relationship with colleagues or with the social environment (Barr, n.d.; Friedmann et al., 2014). Nurses who have worked for more than 5 years will have more experience than nurses who work for less than 5 years (Hegney, Desley G; Craigie, Mark; Hemsworth, 2014; Kaur et al., 2018; S. J. Rn et al., 2017). Nurses who have attended training in accordance with their competencies (Kaur et al., 2018). Compassion satisfaction of nurses is a feeling of pleasure felt by a nurse when able to do a job for people who need care (Beth Hudnall Stamm, 2010). However, compassion satisfaction can be a compassion fatigue which is a negative aspect of helping those who experience traumatic stress and suffering

Several factors are related to the increasing numbers of those with compassion fatigue, including the inability of nurses to control core of selfevaluation and five-factor models (FFM) of personality traits (Barr, 2018), high workload, pressure in work and imbalance between rewards and work (Hämmig, 2018), coping nurses and poor emotional support, rejection, and giving up behavior (Kellogg et al., 2018).

\section{CONCLUSION}

From the results of the study it can be concluded that to get a clinical nurse who has a good professional quality of life value, it is necessary to focus on the things that can influence it. An intervention is needed to reduce and prevent clinical nurses from fatigue and secondary traumatic stress and increase satisfaction compassion to improve high quality care in clinical settings.

\section{REFERENCES}

Amin, A. A., Vankar, J. R., Nimbalkar, S. M., \& Phatak, A. G. (2015). Perceived Stress and Professional Quality of Life in Neonatal Intensive Care Unit Nurses in Gujarat, India. 82(November), 10011005. https://doi.org/10.1007/s12098-0151794-3

Barr, P. (n.d.). The five-factor model of personality, work stress, and professional quality of life in neonatal intensive care unit nurses. 0-2. https://doi.org/10.1111/jan.13543

Barr, P. (2018). Personality Traits, State Positive and Negative Affect, and Professional Quality of Life in Neonatal Nurses. Journal of Obstetric, Gynecologic, \& Neonatal Nursing, (September). https://doi.org/10.1016/j.jogn.2018.08.003

Figley, C. (2018). COMPASSION FATIGUE : Coping with Secondary Traumatic Stress Disorder in Those Who Treat the Traumatized. NY: Brunner / Routledge. (July).

Foster, C. (2019). Investigating professional quality of life in nursing staff working in Adolescent Psychiatric Intensive Care Units ( PICUs ). https://doi.org/10.1108/JMHTEP-04-20180023

Friedmann, E., Mcquillan, K. A., Gilmore, R., Kramer, R. N. B., Bm, B. S., \& Murray, M. (2014). Trauma Nurses. 21(4). https://doi.org/10.1097/JTN.0000000000000 055

Hämmig, 0. (2018). Explaining burnout and the intention to leave the profession among health professionals - a cross-sectional study in a hospital setting in Switzerland. 1-11.

Hegney, Desley G; Craigie, Mark; Hemsworth, D. et al. (2014). Compassion satisfaction, compassion fatigue, anxiety, depression and stress in registered nurses in Australia: study 1 results. (July 2013), 506-518. https://doi.org/10.1111/jonm.12160

Hegney, D. G., Rees, C. S., Eley, R., Osseiran-moisson, R., \& Rees, C. S. (2015). The contribution of individual psychological resilience in determining the professional quality of life of Australian nurses. 6(October). https://doi.org/10.3389/fpsyg.2015.01613

Jang, I., Kim, Y., \& Kim, K. (2016). Professionalism and professional quality of life for oncology nurses. 111. https://doi.org/10.1111/jocn.13330

Kaur, A., Sharma, M. P., \& Chaturvedi, S. K. (2018). Professional Quality of Life among Professional Care Providers at Cancer Palliative Care Centers in Bengaluru , India. 167-172. https://doi.org/10.4103/IJPC.IJPC

Kellogg, M. B., Knight, M., Dowling, J. S., \& Crawford, S. L. (2018). Journal of Pediatric Nursing Secondary Traumatic Stress in Pediatric Nurses is. Journal of Pediatric Nursing, 43, 97-103. https://doi.org/10.1016/j.pedn.2018.08.016

Rn, C. F., \& Rn, M. Y. (2017). Associations of professional quality of life and social support 
with health in clinical nurses. (June), 1-8. https://doi.org/10.1111/jonm.12530

Rn, S. J., Hons, B., Rn, L. P., \& Rn, J. L. (2017). Australian intensive care nurses. Australian Critical Care. https://doi.org/10.1016/j.aucc.2017.10.003

Stamm, 2016. Bibliography, P. M., Reference, S., Bibliography, C., Using, D. S., Measure, P., \& Style, A. P. A. (2016). A Comprehensive Bibliography of Documents Specifically Using the ProQOL Measure. (January).

Stamm, B.H. (2010). Comprehensive Bibliography of The Effect of Caring For Those Who Have Experienced Extremely Stressful Events and
Suffering.

Stamm, Beth Hudnall. (2010). 2010 The Concise ProQOL Manual.

Teffo, M. E., Levin, J., \& Rispel, L. C. (2018). Compassion satisfaction, burnout and secondary traumatic stress among termination of pregnancy providers in two South African provinces. 1-9. https://doi.org/10.1111/jog.13665

Wu, S., Singh-carlson, S., Odell, A., Reynolds, G., \& Su, Y. (2016). Compassion Fatigue, Burnout, and Compassion Satisfaction Among Oncology Nurses in the United States and Canada. 43(4), 161-169. https://doi.org/10.1188/16.0NF.E161-E169 